\title{
OBSERVATIONS OF THE TRIPLE STAR ETA VIRGINIS WITH A LONG BASELINE OPTICAL INTERFEROMETER
}

\author{
C. A. Hummel ${ }^{1}$ \\ RESUMEN
}

Hemos observado la estrella triple jerárquica Eta Virginis con el interferómetro óptico prototipo de US Naval Observatory y presentamos las primeras imágenes con las tres componentes resueltas. Las estrellas del par cerrado tienen una separación entre 4 y 9 milisegundos de arco. Se observaron los movimientos orbitales de estas estrellas durante el invierno y la primavera del 2002. Se han obtenido órbitas astrométricas preliminares de las dos componentes del par cerrado con referencia a la terciaria, lo cual permite estimar el cociente de masas (1.27) del par cerrado. También obtuvimos que la inclinación orbital relativa es de 31 grados. Describimos el proceso de ajuste del modelo a los datos interferométricos y espectroscópicos, y discutimos algunos parámetros astrofísicos de las componentes.

\section{ABSTRACT}

We observed the hierarchical triple star Eta Virginis with the Navy Prototype Optical Interferometer and present the first images resolving all three components. The stars in the close pair have a separation between 4 and 9 milliarcseconds. The orbital motions of the stars were followed during the winter and spring of 2002 . Preliminary, astrometrically determined orbits of the two components in the close pair by reference to the tertiary were derived, thus enabling the estimation of the mass ratio (1.27) of the components in the close pair. We also determined the relative orbital inclination to be 31 degrees. We describe the model-fitting process to interferometric and spectroscopic data, and discuss some astrophysical component parameters.

Key Words: STARS: INDIVIDUAL (ETA VIRGINIS) - STARS: FUNDAMENTAL PARAMETERS

\section{INTRODUCTION}

From a recent paper by Sterzik \& Tokovinin (2002), we learn that there are only 22 stellar triple systems with both orbits determined visually. Eight of these have questionable elements and only three have had their ascending nodes identified using radial velocity measurements. These data were compiled from the MSC data base maintained by A. Tokovinin (1993).

For the study of relative orbit orientations and other elements related to predictions by star formation and dynamical evolution theories, it is obviously necessary to obtain more definite orbital solutions. Here, as in the case of mass determinations for spectroscopic binaries, very high angular resolution is necessary to resolve the close pair in the hierarchical triple visually.

Enter long baseline optical interferometry. This technique of synthesizing large diameter apertures with an array of smaller telescopes has been used to successfully resolve more than two dozen spectroscopic binaries (counting only published results), and has made mass determinations possible for a majority of the components. It is with such an interfer-

\footnotetext{
${ }^{1}$ US Naval Observatory, USA.
}

ometer that we have been able to resolve all three components in the triple star $\eta$ Virginis and determine their visual orbits.

\section{THE TRUTH ABOUT OPTICAL INTERFEROMETERS}

When it comes to high angular resolution at reasonable cost (i.e. not having to build a single telescope 100 meters or larger), there is no alternative to long baseline interferometers. There are two imaging interferometric arrays (NPOI [ftp.nofs.navy.mil/projects/npoi/ and CHARA [www.chara.gsu.edu/CHARA/array.html]) under construction with maximum baseline lengths of more than $300 \mathrm{~m}$, giving angular resolutions better than 1 milliarcsecond (mas). This resolution is needed to resolve most spectroscopic binaries. The trade-off being made by using sparse arrays is with the much reduced imaging capabilities. However, this is not an issue for observing multiple stellar systems.

The complexity of interferometric arrays means that these projects often take more time to become fully operational. The large number of reflections needed to bring the light from the telescopes to the 

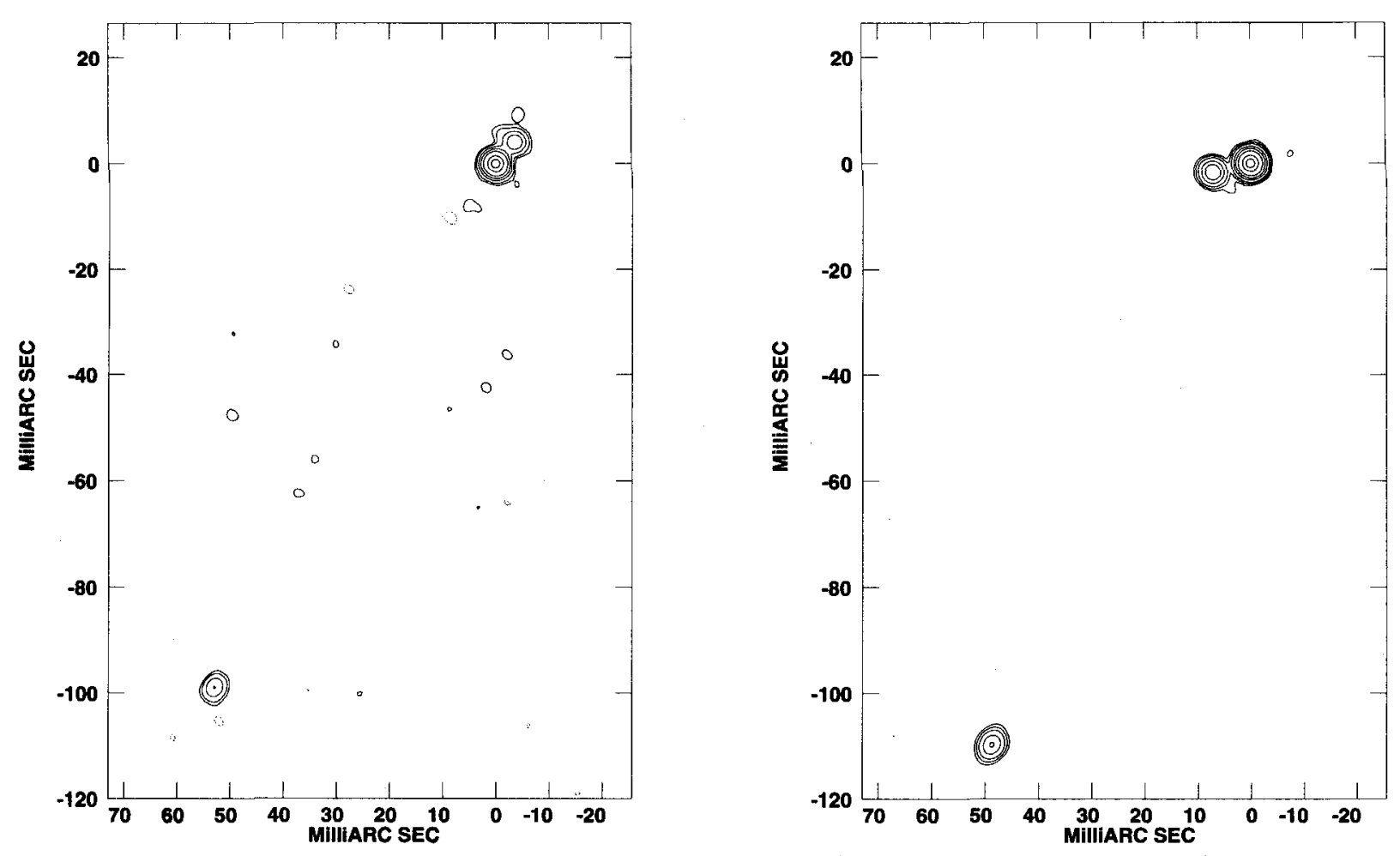

Fig. 1. Images of $\eta$ Virginis for February 15 and May 19 of 2002 . Note that the image scales are the same and that orbital motion in component $\mathrm{B}$ is also detected.

beam combiner has caused the throughput, and consequently the sensitivity, of interferometers to be rather poor compared to even a simple 1-m-class telescope. Also, since interferometers are adaptive optical systems, enough photons must be available to correct for tip and tilt of the wavefront within the coherence time, further degrading the sensitivity. The limiting magnitude of NPOI is currently $V=5$, but is expected to be improved with the installation of beam compressors that will enable the full aperture of $36 \mathrm{~cm}$ to be used, given the diameter of the feed system pipes.

It is in this context that a recent achievement of an interferometric array can be appreciated. Since early 2002, the Navy Prototype Optical Interferometer (NPOI) can combine the light from up to six telescopes simultaneously, providing up to 15 baseline combinations. This greatly enhances the rate at which the synthesized telescope aperture is sampled, and makes it possible to survey a large number of stars for multiplicity.

\section{OBSERVATIONS WITH THE NAVY PROTOTYPE OPTICAL INTERFEROMETER}

The NPOI project was described in detail by Armstrong et al. (1998). The NPOI operates in the optical spectrum, and will achieve a resolution of 200 microarcseconds on its longest baselines of 430 $\mathrm{m}$. The longest baseline currently in use is $64 \mathrm{~m}$, providing a nominal resolution of 1 mas.

The observations of $\eta$ Virginis with the new sixstation beam combiner commenced after this triple system was suggested by A. Tokovinin as a suitable candidate for NPOI. The first night of observations showed that the close pair was easily resolved for the first time on the long baselines, while the components of the wide pair were close enough to be within the interferometric field of view limited by bandwidth-smearing on the shortest baselines. Images obtained at two different epochs using standard interferometric imaging software originally developed for radio VLBI are shown in Fig. 1 and show clearly the orbital motion of both binary components. 


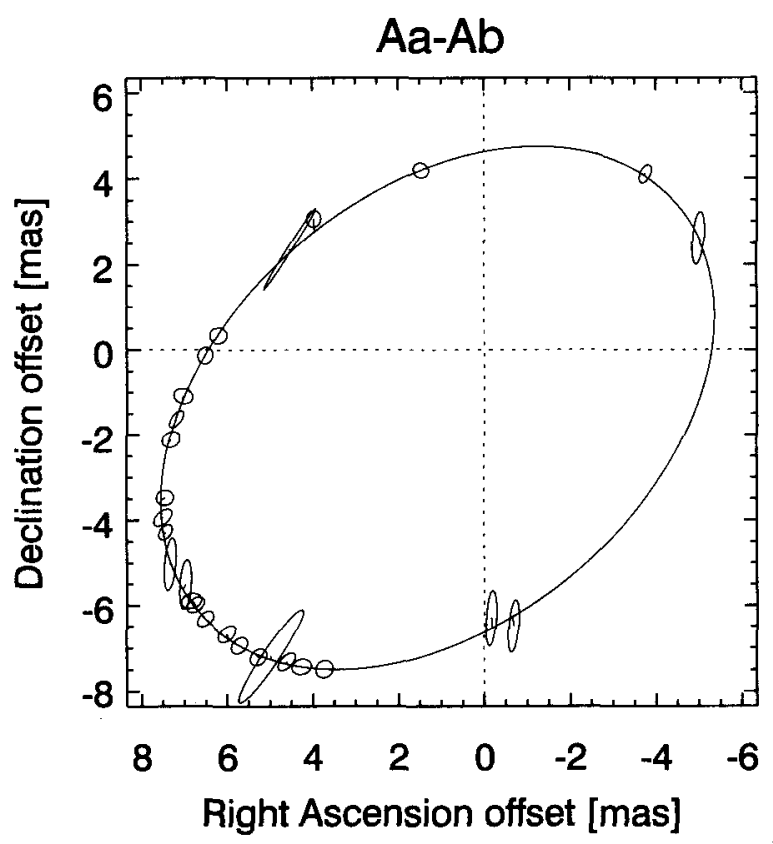

Fig. 2. Apparent orbit of the close pair Aa-Ab.

\section{COMBINED MODELING OF SPECTROSCOPY AND INTERFEROMETRY}

The close pair in $\eta$ Virginis is a double-lined spectroscopic binary with a period of 72 days, while the orbit of the wide pair with a period of $\mathbf{4 7 7 0}$ days has been observed with speckle interferometry. These data have been analyzed and published by Hartkopf et al. (1992), and we combine them with our interferometry to determine the visual orbit of the close pair, as well as absolute magnitudes (via the orbital parallax and total apparent magnitudes) and colors of the individual component stars. The results have recently been published (Hummel et al. 2003). Figure 2 shows the orbit of the close pair.

We determine, with the proper identification of the ascending nodes, the relative orbital inclination to be 31 degrees. In addition, by referencing the position of components $\mathrm{Aa}$ and $\mathrm{Ab}$ to the position of the tertiary component $\mathrm{B}$, their absolute orbits can be determined, thus giving an independent estimate of the mass ratio of components $\mathrm{Aa}$ and $\mathrm{Ab}$. The result is $1.27 \pm 0.09$, to be compared to $1.33 \pm 0.02$ determined from spectroscopy. In our hierarchical model for $\eta$ Virginis, the absolute orbits are encoded in the relative orbit $\mathrm{Aa}-\mathrm{Ab}$ and the orbit of the photocenter of $A$ around its center of mass, causing the apparent motion of component $B$ to occur in a series of loops, as shown in Fig. 3.

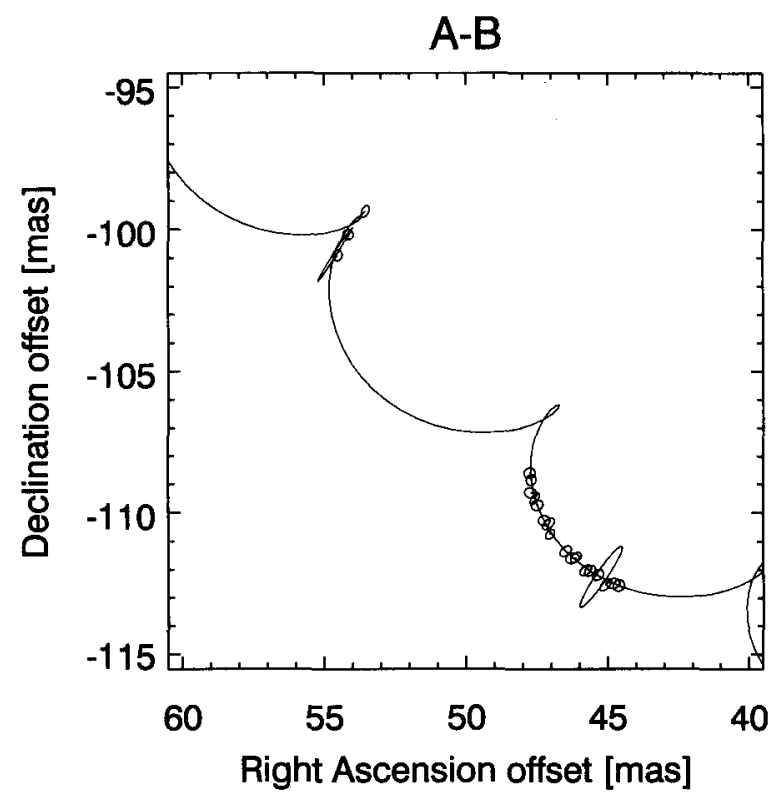

Fig. 3. Detail of the orbit of B around the photocenter of A showing the NPOI measurements. The model loops are caused by the motion of the photocenter around the center of mass of component $\mathrm{A}$.

\section{PHYSICAL COMPONENT PARAMETERS}

Our hierarchical model for the combined data contains, aside from the orbital elements of the two pairs, physical component parameters such as the masses, magnitudes, and angular diameters. Since this model is used for any combination of data from interferometry, astrometry, and spectroscopy, we chose model parameters that are either natural parameters for a particular data set, or (nonexclusive) are physical, such as a mass. Also, no parameter is allowed to be a combination of other parameters in order to avoid inconsistencies. For example, the orbital parallax can be derived from the masses, the semi-major axis in milliarcseconds, and the period and therefore is not a model parameter itself. For a single-lined spectroscopic binary with visual orbit, only the secondary mass is a fit parameter with the understanding that only the mass function is constrained, but not the secondary mass itself.

In this situation, if a parallax could be added to the data set, for example from Hipparcos measurements, it would then constrain both masses individually.

For the components in $\eta$ Virginis we obtain $\mathcal{M}_{\mathrm{A} a} / \mathcal{M}_{\odot}=2.68 \pm 0.15, \mathcal{M}_{\mathrm{A} b} / \mathcal{M}_{\odot}=2.04 \pm 0.10$, and $\mathcal{M}_{\mathrm{B}} / \mathcal{M}_{\odot}=1.66 \pm 0.16$. The apparent visual magnitudes are $4.2,6.0$, and 6.5 , respectively, for spectral types of A2IV, A4V, and A8-F0V. The in- 
dividual component diameters are all unresolved at less than 0.5 mas.

The NPOI is a collaboration between the US Naval Observatory and the Naval Research Laboratory, in association with Lowell Observatory at Flagstaff, Arizona. This work is the result of an effort of the entire NPOI team to make the six-station array work. NPOI is funded by the Office of Naval Research and the Oceanographer of the Navy.

\section{REFERENCES}

Armstrong, J. T. et al. 1998, ApJ, 496, 550

Hartkopf, W. I., McAlister, H. A., Yang, X., \& Fekel, F. C. 1992, AJ, 103, 1976

Hummel, C. A., Benson, J. A., Hutter, D. J., Johnston, K. J., Mozurkewich, D., Armstrong, J. T., Hindsley, R. B., Gilbreath, G. C., Rickard, L. J., \& White, N. M. $2003, A J, 125,2630$

Sterzik, M. F., \& Tokovinin, A. A. 2002, A\&A, 384, 1030

Tokovinin, A. A. 1993, Astron. Lett., 19, 383

\section{DISCUSSION}

Horch - Have you checked the positions of the components on the mass-luminosity relation and are they consistent with what we currently expect?

Hummel - I think we have, and the answer was "yes", but I will double check my notes.

Scarfe - Are all three stars on the main sequence or is there any sign of departure from it?

Hummel - The primary has evolved, but the other components are still on the main sequence.

Guinan - What is the typical precision of your position measures with NPOI?

Hummel - On Mizar A it is $70 \mu$ arcsec, on $\eta$ Vir a little worse because of the calibration.

Christian A. Hummel: US Naval Observatory, 3450 Massachusetts Av NW, Washington, DC 20392, USA (cah@usno.navy.mil). 